\title{
Predictors of Response to Therapy Among Post Thyroidectomy Adult Filipino Patients with Papillary Thyroid Carcinoma Based on the 2015 American Thyroid Association Guidelines*
}

\author{
Aivind Gabrielle Santiago, ${ }^{1}$ Maria Jocelyn Isidro, ${ }^{1}$ Joseph Parra ${ }^{2}$ \\ ${ }^{1}$ Section of Endocrinology, Diabetes and Metabolism, Department of Medicine, Makati Medical Center, Makati City, Philippines \\ ${ }^{2}$ Oncology Unit, Makati Medical Center, Makati City, Philippines
}

\begin{abstract}
Objective. To identify factors independently associated with incomplete response to therapy based on the 2015 ATA guidelines in surgically treated Filipino patients with papillary thyroid carcinoma (PTC).

Methodology. This is a retrospective cohort study of adults aged 21-74 years with papillary thyroid carcinoma (PTC) treated with surgery with or without radioactive iodine therapy (RAI) in Makati Medical Center from 2013 to 2017 . We collected the following factors through a review of charts: age at diagnosis, gender, family history of thyroid cancer, date of surgery, tumor size, capsular/lymphovascular invasion, lymph node/distant metastases, stage, risk of recurrence, dose of post-surgical RAI therapy, initial post-treatment serum Thyroglobulin ( $\mathrm{Tg}$ ) and anti-Tg antibody levels (Negative Tg level: suppressed non-stimulated $\mathrm{Tg}<0.2 \mathrm{ng} / \mathrm{mL}$ or TSH-stimulated $\mathrm{Tg}<1 \mathrm{ng} / \mathrm{mL}$; Positive $\mathrm{Tg}$ level: suppressed $\mathrm{Tg}$ $\geq 1 \mathrm{ng} / \mathrm{mL}$ or a TSH-stimulated $\mathrm{Tg} \geq 10 \mathrm{ng} / \mathrm{mL}$ or rising anti-Tg antibody levels), thyroid stimulating hormone suppression, post-operative imaging studies and levothyroxine dose. Response to therapy was checked 6-24 months post-therapy.

Results. We analyzed a total of 115 patients with PTC who underwent thyroidectomy. Patients who had family history of thyroid cancer were less likely to have an incomplete or indeterminate response $(p=0.045)$. None of the patients with excellent response had lymphovascular invasion. Having a positive $\mathrm{Tg}(p=0.001)$ and positive anti-Tg postoperatively $(p<0.001)$ were strongly associated with incomplete or indeterminate response.
\end{abstract}

Conclusion. Patients who were positive for thyroglobulin and anti-thyroglobulin post-operatively were strongly associated with incomplete or indeterminate response to therapy in PTC.

Key words: papillary thyroid cancer, response to papillary thyroid cancer therapy, well-differentiated thyroid cancer

\section{INTRODUCTION}

The most common endocrine malignancy is welldifferentiated thyroid cancer. ${ }^{1}$ In a report based upon the Surveillance, Epidemiology, and End Results (SEER) database from 1975 to 2012, the incidence of papillary cancer increased from 4.8 to 14.9 per 100,000. Compared to any other malignancies in recent years, the age- and gender-adjusted incidence of thyroid cancer has increased more rapidly. This was observed in both genders and all ethnic backgrounds. ${ }^{2}$

Several studies showed that Filipinos have a higher risk of recurrence rates and mortality in thyroid malignancies.-8 Kus et al., showed that Filipinos in Canada have a significantly increased risk of thyroid cancer recurrence in 5 years at $25 \%$ compared with all other racial/ethnic groups where the recurrence rate was at $9.5 \%$, even after adjusting for confounders. ${ }^{4}$ Lo et al., reported that papillary thyroid cancer (PTC), which is generally known to have a good prognosis, had more aggressive features in cases in the Philippines. Filipino patients are younger, have larger tumor sizes, and had a higher degree of distant metastases and recurrence rate. ${ }^{5}$

According to the 2015 American Thyroid Association (ATA) Guidelines, initial therapy for patients with differentiated thyroid cancer such as PTC should aim to improve survival (overall and disease-specific), reduce the risk of persistent/ recurrent disease and associated morbidity, and accurate disease staging with risk stratification, while minimizing treatment-related complications and unnecessary therapy. The treatment recommendation for PTC is complete surgical resection, with postoperative adjunctive therapy such as radioactive iodine (RAI) and thyroid stimulating hormone (TSH) suppression. The ATA 2015 guidelines classify response to therapy as: (1) excellent response when there is no clinical, biochemical, or structural evidence of 
disease, (2) biochemical incomplete response when there is abnormal thyroglobulin ( $\mathrm{Tg}$ ) or rising anti-thyroglobulin (anti-Tg) antibody levels in the absence of localizable disease, (3) structural incomplete response for persistent or newly identified loco-regional or distant metastases and (4) indeterminate response for nonspecific biochemical or structural findings that cannot be confidently classified as either benign or malignant including patients with stable or declining anti-Tg antibody levels without definitive structural evidence of disease. ${ }^{8}$ Each category has its own predicted clinical outcomes and management implications.

Response to therapy is one of the parameters that can be utilized to assess clinical outcomes of the disease and direct the clinical decision with regards to management and follow-up. This is especially important in Filipino patients, who tend to have a more aggressive course of PTC. Currently, we have little evidence regarding the response to therapy of Filipinos with papillary thyroid cancer using the 2015 ATA Guidelines. This study aims to identify factors independently associated with having an incomplete response to therapy in surgically treated Filipino patients with papillary thyroid cancer.

\section{METHODOLOGY}

The study is a retrospective cohort of all patients with papillary thyroid carcinoma who underwent surgery, with or without radioactive iodine therapy in Makati Medical Center from 2013 to 2017. The Institutional Review Board (IRB) of the hospital approved the study design. The inpatient and outpatient medical records of all adult patients aged at least 18 years old, who underwent thyroidectomy (lobectomy, near total, or total) with or without neck dissection, diagnosed through histopathology as having papillary thyroid carcinoma, with documented response to therapy up to 24 months post-surgery were reviewed and included in the study. No randomization was done because all charts that satisfied the inclusion criteria were included. We cross-referenced medical records, in-hospital cancer registry, and outpatient charts with attending endocrinologists for a comprehensive review of charts.

We collected the following information: age at diagnosis, gender, family history of thyroid cancer, date of surgery, tumor size, capsular or vascular invasion, presence of lymph node or distant metastases, stage, risk of recurrence, dose of post-surgical RAI therapy if done, initial postablative and post-surgical thyroglobulin (Tg) and anti-Tg antibody levels in serum, thyroid stimulating hormone (TSH) suppression, results of imaging studies done postoperatively (ultrasound, RAI whole body scan, CT scan, PET scan) and levothyroxine suppressive dose.

A negative Tg level refers to either a suppressed nonstimulated $\mathrm{Tg}<0.2 \mathrm{ng} / \mathrm{mL}$ or $\mathrm{TSH}$-stimulated $\mathrm{Tg}<1 \mathrm{ng} /$ $\mathrm{mL}$, while a positive thyroglobulin level refers to either a suppressed $\mathrm{Tg} \geq 1 \mathrm{ng} / \mathrm{mL}$ or a TSH-stimulated $\mathrm{Tg} \geq 10 \mathrm{ng} /$ $\mathrm{mL}$ or a rising anti-Tg antibody levels. The assay used for $\mathrm{Tg}$ levels is Immunoradiometric Assay (IRMA).

The primary outcome of the study is the response to therapy after six to 24 months, as defined by the 2015 American Thyroid Association Guidelines. ${ }^{8}$ They were classified as having an (1) excellent response if they had a negative imaging and either a suppressed $\mathrm{Tg}<0.2 \mathrm{ng} / \mathrm{mL}$ or TSH-stimulated $\mathrm{Tg}<1 \mathrm{ng} / \mathrm{mL}$, (2) biochemical incomplete response if they had a negative imaging and suppressed Tg more than or equal to $1 \mathrm{ng} / \mathrm{mL}$ or stimulated Tg more than or equal to $10 \mathrm{ng} / \mathrm{mL}$ or rising anti-Tg antibody levels, (3) structural or functional evidence of disease with any $\mathrm{Tg}$ level with or without anti-Tg antibodies, or (4) indeterminate response for nonspecific findings on imaging studies, faint uptake in the thyroid bed on RAI scanning, detectable non-stimulated Tg but $<1 \mathrm{ng} /$ $\mathrm{mL}$, detectable stimulated $\mathrm{Tg}$ but $<10 \mathrm{ng} / \mathrm{mL}$ or stable or declining anti-Tg antibodies in the absence of structural or functional disease. Patients who did not have RAI as part of their initial therapy were still included and grouped with the same definitions of response to therapy.

We required a minimum sample of 122 patients at $90 \%$ power to detect an odds ratio of 3.674 of multifocal type papillary thyroid carcinoma for an incomplete response in Filipino patients. ${ }^{9}$ Of the 136 records reviewed, 115 satisfied the inclusion criteria. Our sample of 115 was greater than the $80 \%$ power requirement at a minimum of 91 patients. Descriptive statistics were used to summarize the general and clinical characteristics of the patients. Frequency and proportion were used for categorical variables. Shapiro-Wilk test was used to determine the normality of continuous variables. Continuous quantitative data that did not meet the normality assumption were summarized using median and range. Simple and multiple logistic regression analysis was used to determine the factors associated with an incomplete or indeterminate response to therapy post-thyroidectomy. Simple logistic regression was performed to determine whether there was an association between each independent variable and the dependent variable (incomplete/indeterminate response), before adjusting for other factors. Levothyroxine intake, TNM stage, tumor size, surgical margins, extrathyroidal extension, TSH level, thyroglobulin, anti-thyroglobulin and RAI were all included and analyzed in the multiple regression analysis due to their relevance in the AMES, ATA and MACIS criteria. The variables were adjusted by age, sex, and family history of thyroid cancer. Crude and adjusted odds ratios and corresponding 95\% confidence intervals were reported. Missing observations were not imputed. Null hypothesis was rejected at $0.05 \alpha$-level of significance. STATA 15.0 (StateCorp SE, College Station, TX, USA) was used for data analysis (Figure 1).

\section{RESULTS}

We analyzed a total of 115 patients with papillary thyroid carcinoma who underwent thyroidectomy (Table 1). Overall, there were $68(59 \%)$ with excellent response. Biochemical incomplete response was observed in 12 $(10 \%)$, structural incomplete response in $30(26 \%)$, and

Table 1. Response to therapy among post-thyroidectomy adult Filipinos patients with PTC $(n=115)$

\begin{tabular}{lccc}
\hline & Total $(\mathbf{n}=\mathbf{1 1 5})$ & RAI + (n=90) & RAI - (n=25) \\
\cline { 2 - 4 } & \multicolumn{3}{c}{ Frequency (\%) } \\
\hline Excellent response & $68(59.13 \%)$ & $51(56.67 \%)$ & $17(68 \%)$ \\
Biochemical incomplete & $12(10.43 \%)$ & $12(13.33 \%)$ & $0(0 \%)$ \\
Structural incomplete & $30(26.09 \%)$ & $23(25.56 \%)$ & $7(28 \%)$ \\
Indeterminate response & $5(4.35 \%)$ & $4(4.44 \%)$ & $1(4 \%)$ \\
\hline & &
\end{tabular}




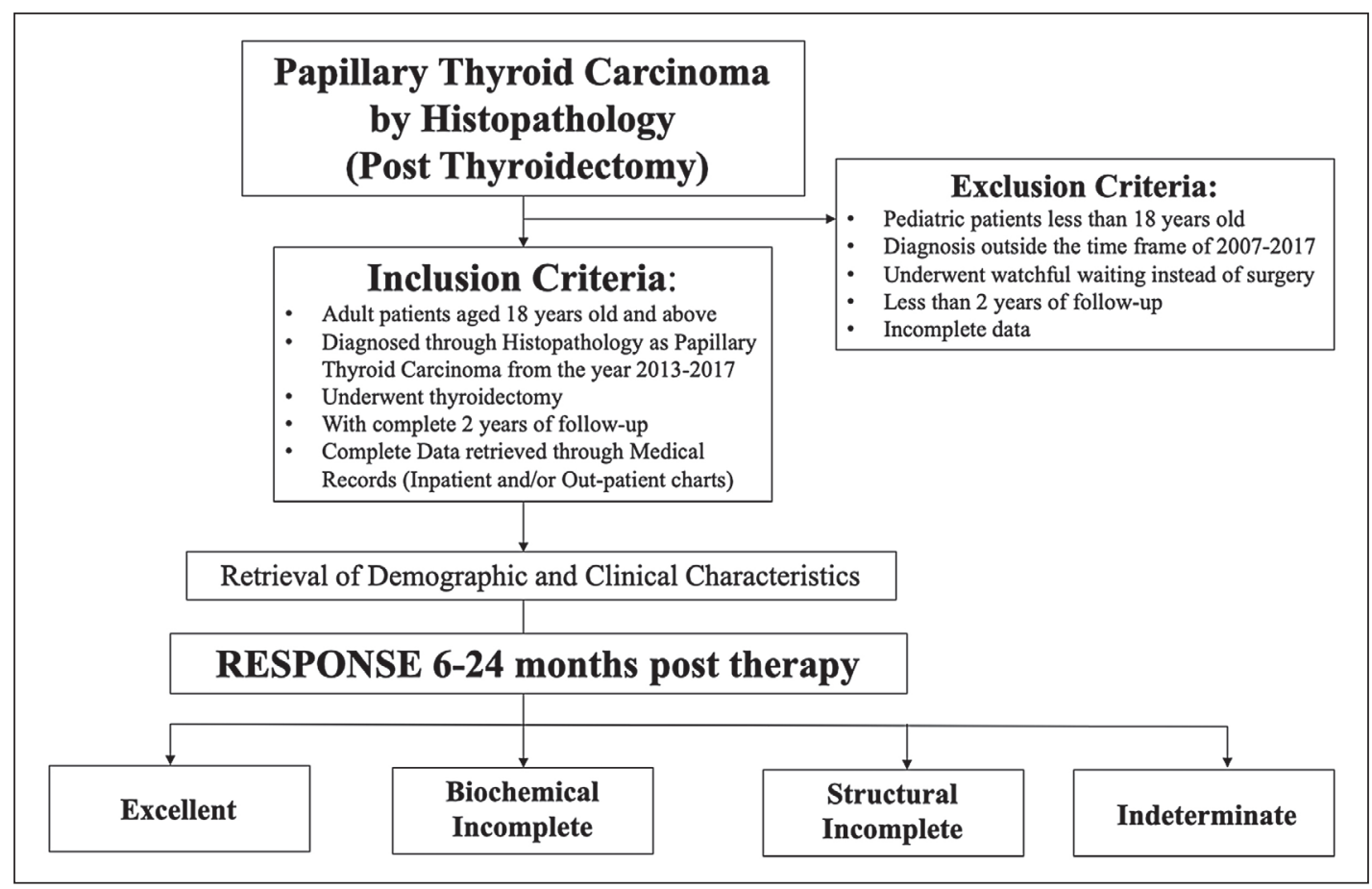

Figure 1. Study flowchart for data collection.

indeterminate response in five patients. When grouped according to RAI therapy, there were 90 (78\%) who had received RAI. Among patients who received RAI, excellent response was observed in $51(57 \%)$ patients. Among those who did not receive RAI, there were 17 (68\%) who had an excellent response.

Comparing groups according to response type, the excellent response group had a higher proportion of patients who had reported having a family history of thyroid cancer (23.53\% versus $8.51 \%, p=0.037)$, and none of them had lymphovascular invasion ( $0 \%$ versus $15 \%, p=0.001)$. The group who did not have an excellent response had a higher proportion of initially positive post-operative results for thyroglobulin $(p<0.001)$ and anti-Tg $(p=<0.001)$.

A higher proportion of high-risk patients on AMES and MACIS had incomplete or indeterminate responses (Table 2), but this was not statistically significant. On ATA, 53\% of those with incomplete or indeterminate responses were classified as high risk, which was significantly higher than the excellent response group $(p<0.001)$.

Patients who reported that they had a family history of thyroid cancer were approximately $70 \%$ less likely to have an incomplete or indeterminate response (cOR 0.302, 95\% CI 0.09 to $0.97, p=0.045$ ). Having a positive Tg (aOR 121.88, 95\% CI 7.74 to $1918.46, p=0.001$ ) and positive anti-Tg postoperatively (aOR 46.61, 95\% CI 7.36 to $295.05, p<0.001$ ) were strongly associated with incomplete or indeterminate response, even after performing multiple logistic regression to adjust for age, sex, and family histo hyroid cancer (Table 3).

\section{DISCUSSION}

The four responses to therapy were coined by Tuttle et al., and were utilized by the 2015 American Thyroid
Association to assess the clinical status of patients with well-differentiated thyroid cancer post-therapy (total thyroidectomy and radioactive remnant ablation). ${ }^{10}$ The four classifications of treatment responses namely: excellent, biochemical incomplete, structural incomplete and indeterminate have different clinical outcomes.

Patients classified as having excellent response had 1-4\% recurrence, with only $<1 \%$ disease specific death. Thirty percent of patients with biochemical incomplete response spontaneously evolved to No Evidence of Disease (NED), $20 \%$ achieved NED after additional therapy, 20\% developed structural disease, and $<1 \%$ had disease specific death.

According to the ATA, for structural incomplete response, $50-80 \%$ of patients continue to have persistent disease despite additional therapy, disease specific death rates at $11 \%$ with locoregional metastases and $50 \%$ with distant metastases. $^{8}$

Patients with indeterminate response had $15-20 \%$ structural disease, $<1 \%$ had disease specific death and the rest had resolved or stable disease.

Because of the clinical outcomes that each response represents, it impacts the type of management for each treatment response. A patient with excellent response to therapy is expected to have less intense follow-up and less TSH suppression. In biochemical incomplete response, monitor serum thyroglobulin and anti-thyroglobulin levels to determine the appropriate levothyroxine dose for TSH suppression, any additional tests and therapy, if indicated. Patients with structurally incomplete response usually need additional treatment modalities depending on the findings. In our study, the patients who did not receive RAI but had structurally incomplete response had either positive lymph nodes, metastatic tissue (bone or lung) or recurrence on the thyroid bed. Lastly, in 
Table 2. Demographic and clinical characteristics of adult Filipino patients with papillary thyroid carcinoma $(n=115)$

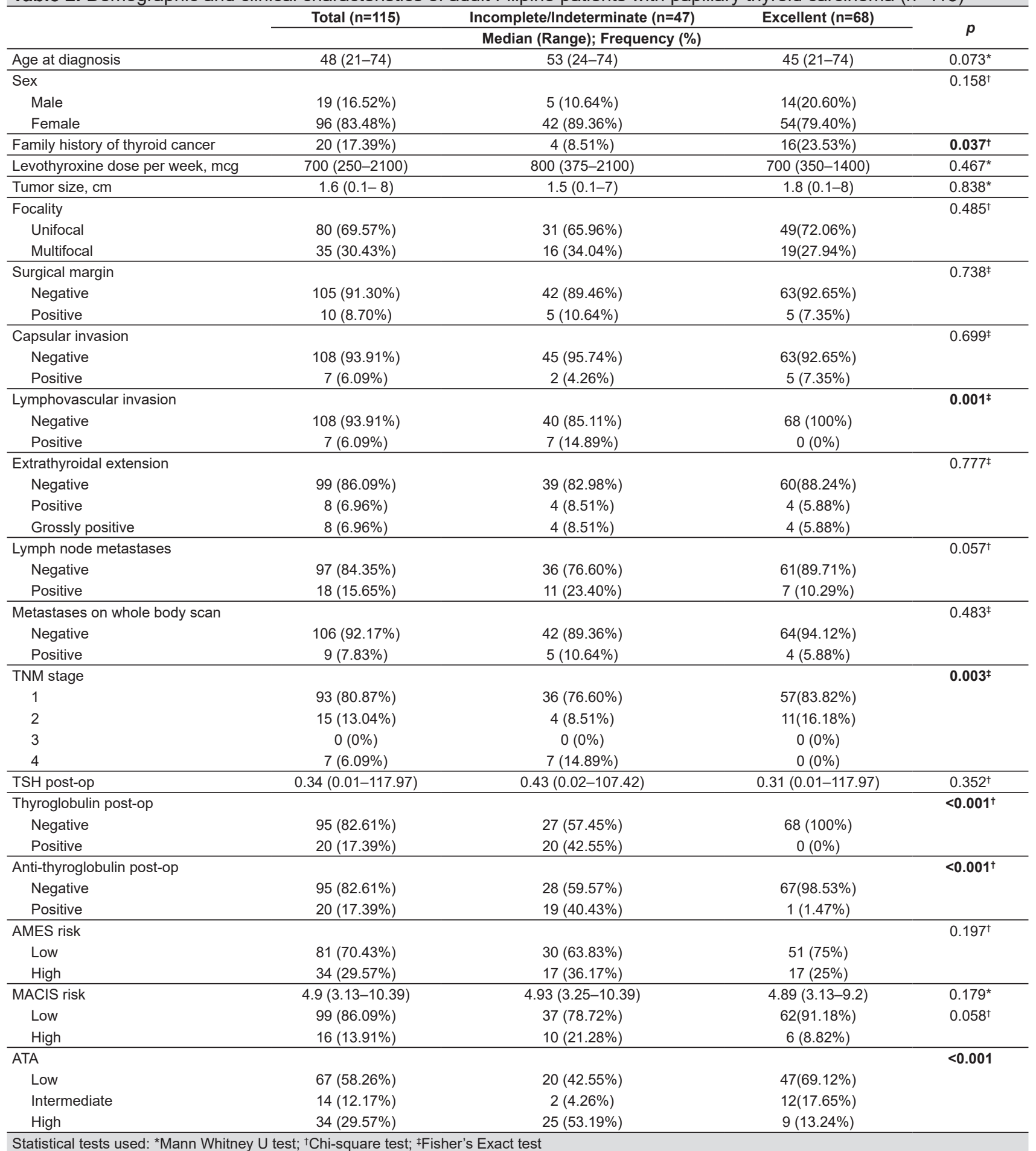

patients with indeterminate response, they are continually observed with appropriate biochemical (thyroglobulin, anti-thyroglobulin) and serial imaging tests; biopsy can be offered for suspicious looking lesions as well. ${ }^{8}$

This study had a total of 115 patients, majority of which are females $(83.48 \%)$ with a mean age of 48 at diagnosis and have negative family history $(17.39 \%)$. This is compatible with the usual profile of patients with PTC as characterized by previous studies. ${ }^{11-13}$ Majority had an excellent response, as expected, as PTC is a disease with a a generally good prognosis. We looked at some of the factors that might affect the patient's response to therapy such as age, gender, family history, tumor size, surgical margin, capsular invasion, extrathyroidal extension, lymph node and distant metastases. A more advanced age was predisposed to having an incomplete response to therapy - this maybe due to a long duration of disease prior to diagnosis. However, the value was not significant.

Initial findings suggested female gender did not have an excellent response as expected, more commonly male gender is a poor prognostic indicator. However, upon further analysis, this was not found to be significant 
Table 3. Predictors of incomplete or indeterminate response $(n=115)$

\begin{tabular}{|c|c|c|c|c|}
\hline & Crude Odds Ratio $(95 \% \mathrm{Cl})$ & $p$ & Adjusted Odds Ratio $(95 \% \mathrm{Cl})$ & $p$ \\
\hline Age at diagnosis & $1.025(0.997-1.05)$ & 0.078 & & \\
\hline \multicolumn{5}{|l|}{ Sex } \\
\hline Male & Reference & - & & \\
\hline Female & $2.178(0.73-6.53)$ & 0.165 & & \\
\hline Family history of thyroid cancer & $0.302(0.09-0.97)$ & 0.045 & & \\
\hline Levothyroxine dose per week, mcg & $1.001(0.999-1.002)$ & 0.461 & $1.001(0.999-1.004)$ & .244 \\
\hline Tumor size, cm & $0.989(0.79-1.24)$ & 0.926 & $1.022(0.66-1.57)$ & .922 \\
\hline \multicolumn{5}{|l|}{ Focality } \\
\hline Unifocal & Reference & - & & \\
\hline Multifocal & $1.331(0.60-2.97)$ & 0.485 & & \\
\hline \multicolumn{5}{|l|}{ Surgical margin } \\
\hline Negative & Reference & - & Reference & - \\
\hline Positive & $1.5(0.41-5.50)$ & 0.541 & $0.663(0.02-24.03)$ & .823 \\
\hline \multicolumn{5}{|l|}{ Capsular invasion } \\
\hline Negative & Reference & - & & \\
\hline Positive & $0.56(0.10-3.02)$ & 0.500 & & \\
\hline \multicolumn{5}{|l|}{ Extrathyroidal extension } \\
\hline Negative & Reference & - & Reference & - \\
\hline Positive/grossly positive & $1.538(0.53-4.44)$ & 0.426 & $1.380(0.32-6.04)$ & .669 \\
\hline \multicolumn{5}{|l|}{ Lymph node metastases } \\
\hline Negative & Reference & - & Reference & - \\
\hline Positive & $2.663(0.95-7.48)$ & 0.063 & $0.697(0.06-8.77)$ & .780 \\
\hline \multicolumn{5}{|l|}{ Metastases on whole body scan } \\
\hline Negative & Reference & - & Reference & - \\
\hline Positive & $1.905(0.48-7.50)$ & 0.357 & $2.131(0.27-17.12)$ & .477 \\
\hline TSH post-op & $0.999(0.98-1.01)$ & 0.863 & $0.990(0.96-1.03)$ & .579 \\
\hline $\mathrm{RAl+}$ & $1.625(0.64-4.15)$ & 0.310 & $1.713(0.27-10.68)$ & .564 \\
\hline Positive thyroglobulin post-op & $102.127(5.97-1748.14)$ & .001 & $121.886(7.74-1918.46)$ & .001 \\
\hline Positive anti-thyroglobulin post-op & $30.789(5.53-171.51)$ & $<.001$ & $46.611(7.36-295.05)$ & $<.001$ \\
\hline
\end{tabular}

probably because the number of females in the study far outnumbered males.

Initially, having a family history significantly showed an association with having an excellent response to therapy. Patients with a family history are likely to be more vigilant and consult much earlier with timely diagnosis and treatment. Upon multiple regression analysis however, the finding did not reach statistical significance.

The presence of lymphovascular invasion in the study equated to an incomplete response, which was true for 7 patients. Lymph node metastasis is the most common route of spread for papillary thyroid carcinoma, but its prognostic implication in several studies is conflicting. ${ }^{14}$ Multifocality, lymphovascular invasion, absence of tumor capsule and extrathyroidal extension are multiple factors reported as predictors of nodal disease. ${ }^{14}$ This finding might change the outlook of clinicians regarding the response to therapy in those with positive lymph node metastases. Tumor size, focality, capsular invasion as well as extrathyroidal extension did not appear to be significant predictors of response in this study.

A positive thyroglobulin (aOR 121.88, 95\% CI 7.74 to 1918.46, $p=0.001$ ) and positive anti-Tg post-operatively (aOR 46.61, 95\% CI 7.36 to $295.05, p<0.001$ ) were strongly associated with incomplete or indeterminate response, even after performing multiple logistic regression to adjust for age, sex, and family history of thyroid cancer. A study by Matthews et al., showed an increase in relative risk of developing recurrence of papillary thyroid cancer if the serum thyroglobulin at the time of RAI is greater than $27.5 \mathrm{ug} / \mathrm{L}$, with a positive predictive value of $31.3 \%{ }^{15}$ Another study by Spencer showed a positive predictive value of $23.1 \%$ if the thyroglobulin level is 2 ug/L or higher. ${ }^{16}$ However, prescribing a cut-off value for serum thyroglobulin level at the time of RAI that would reach clinical prognostic difference is difficult due to the heterogeneity of thyroglobulin assays, and other factors that may affect $\mathrm{Tg}$ levels such as positive anti-Tg and residual normal thyroid tissue. ${ }^{15}$ The Tg level cut-off used in this study for being positive is a suppressed $\mathrm{Tg}$ of $\geq 1 \mathrm{ng} /$ $\mathrm{mL}$, a TSH-stimulated $\mathrm{Tg}$ of $\geq 10 \mathrm{ng} / \mathrm{mL}$, or a rising anti$\mathrm{Tg}$ antibody level as defined by ATA for biochemically incomplete response.

Based on the different risk scores utilized in the study, there is a higher proportion of patients classified as high risk on AMES and MACIS that had incomplete response but this was not statistically significant. However, when the $8^{\text {th }}$ edition of AJCC/TNM staging and ATA 2009 risk stratification were used, there is significant difference between the 2 groups. ${ }^{8}$ The patients with a higher TNM stage and classified as high risk in the ATA guidelines had incomplete response to therapy.

A limitation of the study is that the data collected were highly dependent on the records and charts retrieved. The parameters used in categorizing the patients' responses to therapy were dependent on facilities in which laboratory tests and imaging were available with accurate interpretation and reading. Also, there were 25 patients who were included in the study but did not undergo RAI, and were still classified and grouped according to the ATA response post-therapy. 
The study by Matthews et al., and Spencer noted that the measurement of thyroglobulin level still plays a significant role even when taken at the time of RAI. ${ }^{15-16}$

Although there were a number of factors analyzed, another limitation is the small sample size. The level of significance was reached, but with a wide confidence interval.

The authors acknowledge that the sample size was calculated based on a variables already known to be strongly linked to recurrence rather than a factor that had the least association but could potentially have a biologically meaningful association. We recommend a similar multicenter prospective study, with a larger cohort of patients, that can more accurately identify the factors associated with poor responses to treatment among Filipino patients who will undergo thyroidectomy with or without RAI for papillary thyroid cancer.

A study done by Espiritu et al., among Filipino patients with papillary thyroid cancer showed the presence of BRAF V600E mutation in $70 \%$ of the study population. This finding may explain the difference in the behavior and course of the disease in this selected population. ${ }^{17}$ The inclusion of such cytogenetic tests as an additional parameter to assess the prognosis of patients with PTC post therapy is recommended in future studies.

\section{CONCLUSION}

In conclusion, patients who were positive for thyroglobulin and anti-thyroglobulin post-operatively were strongly associated with incomplete or indeterminate response to therapy in papillary thyroid cancer. However, we recommend to have our results validated in a larger cohort of patients with papillary thyroid cancer.

\section{Statement of Authorship}

All authors certified fulfillment of ICMJE authorship criteria.

\section{Author Disclosure}

The authors declared no conflict of interest.

\section{Funding Source}

None.

\section{References}

1. Alvarado R, Sywak MS, Delbridge L, Sidhu SB. Central lymph node dissection as a secondary procedure for papillary thyroid cancer: Is there added morbidity? Surgery. 2009;145(5):514-8. PMID: 19375610. https://doi.org/10.1016/j.surg.2009.01.013.

2. Ries LAG, Melbert D, Krapcho M, et al (eds). SEER Cancer Statistics Review, 1975-2004. National Cancer Institute. Bethesda, MD. https:// seer.cancer.gov/csr/1975_2004/, based on November 2006 SEER data submission, posted to the SEER web site, 2007.
3. Haselkorn T, Bernstein L, Preston-Martin S, Cozen W, Mack WJ. Descriptive epidemiology of thyroid cancer in Los Angeles County, 1972-1995. Cancer Causes Control. 2000;11(2):163-70. PMID: 10710201. https://doi.org/10.1023/a:1008932123830.

4. Kus LH, Shah M, Eski S, Walfish PG, Freeman JL. Thyroid cancer outcomes in Filipino patients. Arch Otolaryngol Head Neck Surg. 2010;136(2):138-42. PMID: 20157058. https://doi.org/10.1001/ archoto.2009.206.

5. Lo TE, Uy AT, Maningat PD. Well-differentiated thyroid cancer: The Philippine General Hospital experience. Endocrinol Metab (Seoul). 2016;31(1):72-9. PMID: 26754584. PMCID: PMC4803565. https://doi.org/10.3803/EnM.2016.31.1.72.

6. Horn-Ross PL, McClure LA, Chang ET, et al. Papillary thyroid cancer incidence rates vary significantly by birthplace in Asian American women. Cancer Causes Control. 2011;22(3):479-85. PMID: 21207130. PMCID: PMC3291661. https://doi.org/10.1007/s10552-010-9720-5.

7. Gomez SL, Noone AM, Lichtensztajn DY, et al. Cancer incidence trends among Asian American populations in the United States, 1990-2008. J Natl Cancer Inst. 2013;105(15):1096-110. PMID: 23878350. PMCID: PMC3735462. https://doi.org/10.1093/jnci/djt157.

8. Haugen BR, Alexander EK, Bible KC, et al. 2015 American Thyroid Association management guidelines for adult patients with thyroid nodules and differentiated thyroid cancer: The American Thyroid Association guidelines task force on thyroid nodules and differentiated thyroid cancer. Thyroid. 2016;26(1):1-133. PMID: 26462967. PMCID: PMC4739132. https://doi.org/10.1089/thy.2015.0020.

9. Mendoza ES, Lopez AA, Valdez VAU, et al. Predictors of incomplete response to therapy among Filipino patients with papillary thyroid cancer in a tertiary hospital. J Endocrinol Invest. 2016; 39(1):55-62. PMID: 26036600. https://doi.org/10.1007/s40618-015-0319-2.

10. Tuttle RM, Tala H, Shah J, et al. Estimating risk of recurrence in differentiated thyroid cancer after total thyroidectomy and radioactive iodine remnant ablation: Using response to therapy variables to modify the initial risk estimates predicted by the new American Thyroid Association staging system. Thyroid. 20(12):1341-9. PMID: 21034228. PMCID: PMC4845674. https://doi.org/10.1089/thy.2010.0178.

11. Amin A, Badwey A, El-Fatah S. Differentiated thyroid carcinoma: An analysis of 249 patients undergoing therapy and aftercare at a single institution. Clin Nucl Med. 2014;39(2):142- 6. PMID: 24368533. https://doi.org/10.1097/RLU.0000000000000324.

12. Sciuto R, Romano L, Rea S, Marandino F, Sperduti I, Maini CL. Natural history and clinical outcome of differentiated thyroid carcinoma: A retrospective analysis of 1503 patients treated at a single institution. Ann Oncol. 2009;20(10):1728-35. PMID: 19773250. https://doi. org/10.1093/annonc/mdp050.

13. Palme CE, Waseem Z, Raza SN, Eski S, Walfish P, Freeman JL. Management and outcome of recurrent well-differentiated thyroid carcinoma. Arch Otolaryngol Head Neck Surg. 2004;130(7):819-24. PMID: 15262757. https://doi.org/10.1001/archotol.130.7.819.

14. Wang LY, Ganly I. Nodal metastases in thyroid cancer: Prognostic implications and management. Future Oncol. 2016; 12(7): 981-94. PMID: 26948758. PMCID: PMC4992997. https://doi.org/10.2217/ fon.16.10.

15. Matthews TJ, Chua E, Gargya A, Clark J, Gao K, Elliot M. Elevated serum thyroglobulin levels at the time of ablative radioactive iodine therapy indicate a worse prognosis in thyroid cancer: An Australian retrospective cohort study. J Laryngol Otol. 2016;130(Suppl. S4) S50-3. PMID: 27488338. https://doi.org/10.1017/S0022215116008331.

16. Spencer CA. Challenges of serum thyroglobulin $(\mathrm{Tg})$ measurement in the presence of $\mathrm{Tg}$ autoantibodies. J Clin Endocrinol Metab. 2004;89(8):3702-4. PMID: 15292292. https://doi.org/10.1210/jc.20040986.

17. Espiritu GAM, Malana JT, Dumasis AJGV, Ang DC. High preponderance of BRAF V600E mutation in papillary thyroid carcinoma among Filipinos: A clinicopathologic study. J Glob Oncol. 2019;5:1-6. PMID: 30694737. PMCID: PMC6426509. https://doi. org/10.1200/JGO.18.00085.

Authors are required to accomplish, sign and submit scanned copies of the JAFES Author Form consisting of: (1) Authorship Certification, that authors contributed substantially to the work, that the manuscript has been read and approved by all authors, and that the requirements for authorship have been met by each author; (2) the Author Declaration, that the article represents original material that is not being considered for publication or has not been published or accepted for publication elsewhere, that the article does not infringe or violate any copyrights or intellectual property rights, and that no references have been made to predatory/suspected predatory journals; (3) the Author Contribution Disclosure, which lists the specific contributions of authors; (4) the Author Publishing Agreement which retains author copyright, grants publishing and distribution rights to JAFES, and allows JAFES to apply and enforce an Attribution-Non-Commercial Creative Commons user license; and (5) the Conversion to Visual Abstracts (*optional for original articles only) to improve dissemination to practitioners and lay readers Authors are also required to accomplish, sign, and submit the signed ICMJE form for Disclosure of Potential Conflicts of Interest. For original articles, authors are required to submit a scanned copy of the Ethics Review Approval of their research as well as registration in trial registries as appropriate. For manuscripts reporting data from studies involving animals, authors are required to submit a scanned copy of the Institutional Animal Care and Use Committee approval. For Case Reports or Series, and Images in Endocrinology, consent forms, are required for the publication of information about patients; otherwise, appropriate ethical clearance has been obtained from the institutional review board. Articles and any other material published in the JAFES represent the work of the author(s) and should not be construed to reflect the opinions of the Editors or the Publisher. 